\title{
Combining olfactory test and motion analysis sensors in Parkinson's disease preclinical diagnosis: a pilot study
}

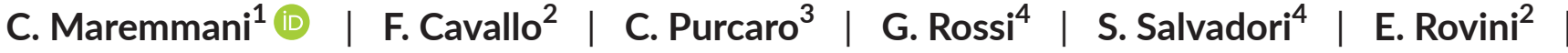

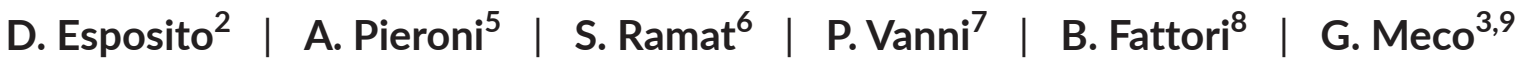

${ }^{1}$ Unità Operativa di Neurologia, Ospedale delle Apuane, Azienda USL Toscana Nord Ovest, Massa, Italy

${ }^{2}$ Istituto di Biorobotica, Scuola Superiore Sant'Anna, Pisa, Italy

${ }^{3}$ Dipartimento di Neurologia e Psichiatria, e Centro di Ricerca per le Malattie Sociali (CIMS), "Sapienza" Università degli Studi di Roma, Roma, Italy

${ }^{4}$ Unità di Epidemiologia e Biostatistica, Istituto di Fisiologia Clinica, Consiglio Nazionale delle Ricerche Area della Ricerca di Pisa, Pisa, Italy

${ }^{5}$ Educazione alla Salute, Azienda USL Toscana Nord Ovest, Carrara, Italy

${ }^{6}$ Dipartimento di Neuroscienze, Azienda Ospedaliero Universitaria Careggi, Firenze, Italy

${ }^{7}$ Unità Operativa di Neurologia, Ospedale Santa Maria Annunziata, Azienda USL Toscana Centro, Firenze, Italy

${ }^{8}$ Unità di Audiologia e Foniatria, Medicina Interna e Sperimentale, Università degli Studi di Pisa, Pisa, Italy

${ }^{9} \mathrm{NCL}$ [Neurological Centre of Latium (Gruppo NEUROMED)] Centro Studi Clinici Malattia di Parkinson, Roma, Italy

\section{Correspondence}

C. Maremmani, Unità Operativa di Neurologia, Ospedale delle Apuane, Azienda USL Toscana Nord Ovest, Massa, Italy.

Email: carlo.maremmani@usInordovest. toscana.it, maremmanicarlo@outlook.it
Objectives: Preclinical diagnosis of Parkinson's disease (PD) is nowadays a topic of interest as the neuropathological process could begin years before the appearance of motor symptoms. Several symptoms, among them hyposmia, could precede motor features in PD. In the preclinical phase of PD, a subclinical reduction in motor skills is highly likely. In this pilot study, we investigate a step-by-step method to achieve preclinical PD diagnosis.

Material and methods: We used the IOIT (Italian Olfactory Identification Test) to screen a population of healthy subjects. We identified 20 subjects with idiopathic hyposmia. Hyposmic subjects underwent an evaluation of motor skills, at baseline and after 1 year, using motion analysis sensors previously created by us.

Results: One subject showed significant worsening in motor measurements. In this subject, we further conducted a dopaminergic challenge test monitored with the same sensors and, finally, he underwent $\left[{ }^{123} \mathrm{l}\right]-\mathrm{FP} / \mathrm{CIT}$ (DaTscan) SPECT brain imaging. The results show that he is probably affected by preclinical PD.

Conclusions: Our pilot study suggests that the combined use of an olfactory test and motor sensors for motion analysis could be useful for a screening of healthy subjects to identify those at a high risk of developing PD.

\section{KEYWORDS}

Italian Olfactory Identification Test, Parkinson's disease, Parkinson's disease preclinical diagnosis, preclinical Parkinson's disease, smell identification test, wearable sensors

\section{1 | INTRODUCTION}

It is widely accepted that the neuropathological process of Parkinson's disease $(\mathrm{PD})$ begins years before the appearance of motor signs. The preclinical phase of PD is believed to last 5-8 years during which the neuropathological process has begun, but there is no clinical evidence of motor disturbances (tremor, bradykinesia, rigidity). Therefore, preclinical diagnosis of PD could be very important to test agents that could be truly neuroprotective and to better understand pathological history of PD. ${ }^{1,2}$
Definite criteria for preclinical diagnosis of PD are still lacking, so this is a topic of great interest and in constant evolution. ${ }^{3,4}$

The diagnosis of PD rests mainly on clinical criteria, although sometimes functional imaging of the nigrostriatal dopaminergic system could be useful (ie single-photon emission computed tomography-SPECT using $\left[{ }^{123} \mathrm{I}\right]$-FP/CIT, a ligand of presynaptic dopamine transporter-DAT). Nevertheless, to achieve preclinical diagnosis, the use of such techniques seems to be mandatory, to confirm a subclinical dysfunction of the nigrostriatal pathway. ${ }^{2-5}$ 
Hyposmia is one of the symptoms that very often precede the appearance of cardinal motor symptoms of PD. Despite its high sensitivity, the specificity of hyposmia in predicting PD is quite low. ${ }^{5}$ For this reason, hypothesizing a mass screening to achieve preclinical diagnosis, combining detection of olfaction with [ $\left.{ }^{123} \mathrm{I}\right]-\mathrm{FP} / \mathrm{CIT}$ SPECT seems impractical, considering the cost and the invasiveness of the latter. ${ }^{5}$ For this reason, there is an unmet need to find low-cost non-invasive screening techniques that could discriminate, among subjects with idiopathic hyposmia, those with a probable underlying initial neurodegenerative process, who could possibly undergo $\left[{ }^{123} \mathrm{I}\right]-\mathrm{FP} / \mathrm{CIT}$ SPECT brain imaging. ${ }^{5}$

In preclinical PD, there could be a progressive or step-like reduction in motor performances until the disease becomes clinically evident. This subclinical reduction in motor abilities might not be perceived by the patient nor by the clinician.

Wearable devices for motion analysis ${ }^{6-10}$ could be useful in detecting a subclinical reduction in motor skills.

We presume that by studying a population of idiopathic hyposmia with this type of sensors, ${ }^{6,7}$ we could find a subgroup of individuals that worsen in motor performances in time. In this subgroup of hyposmic individuals, we also could expect a response to dopaminergic drug challenge using our wearable devices to monitor the changes in motion parameters. We also think that many individuals in this subgroup should have positive results in $\left[{ }^{123} \mathrm{I}\right]-\mathrm{FP} / \mathrm{CIT}$ SPECT brain imaging.

Given these hypotheses, we did a pilot study and here we show the results.

\section{2 | MATERIALS AND METHODS}

A population of healthy subjects aged 60-69 was recruited and underwent an idiopathic hyposmia screening starting with a standardized smell test. With this first step, we identified 20 idiopathic hyposmic subjects of 287 healthy subjects.

Exclusion criteria in recruitment were as follows: a MMSE ${ }^{11}$ score $<27$; history of previous concussive head trauma; previous or present neurological or psychiatric disease; major otolaryngological, orthopaedic, rheumatological or oncological diseases; major metabolic diseases; hyposmia with known aetiology.

Olfaction was tested with IOIT. ${ }^{12}$ Individuals with an IOIT score $\geq 7$ were considered hyposmic and underwent neurological examination, an otolaryngological visit, and a basal magnetic resonance imaging (MRI) scan of the brain. We did not consider minimal vascular lesions in MRI as an exclusion criterion. Hyposmia was considered idiopathic if there was no known cause and if none of the exclusion criteria were present.

During the first evaluation, subjects with idiopathic hyposmia underwent a measurement of 4 motor tasks using inertial sensors suitable for the study of upper and lower limb movements. ${ }^{6,7}$

Motor parameters evaluated: (i) number of thumb-middle finger tappings (THMF); (ii) number of movements in hand opening-closing task (OPCL); (iii) number of movements in hand prono-supination task (PSUP); (iv) variability of tiptoe tapping task (TTFCV) calculated as follows: (maximum frequency - minimum frequency)/maximum frequency $\times 100$.

Each of the previous tasks was executed for 10 seconds 3 times each, measuring all of them. The results were the mean of the 3 measurements.

One year after recruitment, each subject with idiopathic hyposmia underwent neurological examination and measurement of motor performances with the same sensors again. We evaluated each subject for the possible appearance of neurological signs, and we considered the per cent variation of motor performances between the 2 measurements, at 1 year of distance.

Given the absence of normative data and cut-off values for motor measurements, in this study we decided to consider every subject with idiopathic hyposmia that we assessed as a reference point for himself in the motor measurements conducted in time.

For the aim of our study, a worsening that was not due to drugs, neurological diseases or other pathological conditions that could change motor skills and joints functions were considered relevant.

To choose the degree of subclinical worsening that could imply a preclinical PD, we deduced from clinical experience a percentage worsening of motor measurements beyond which preclinical PD was suspected. We considered a subclinical idiopathic worsening greater than $15 \%$ in at least 2 motor tasks as a warning sign for preclinical PD.

The clinical reasoning which prompted us to choose this cut-off value is the following: in patients with PD, Horváth et al ${ }^{13}$ estimated the minimal clinically important difference (MICD) in worsening (mean PD patients' MDS-UPDRS III score $=40.7$ and threshold for MCID to detecting worsening +4.63 points) which, expressed in percentage, corresponds to the $11.38 \%$. Having this in mind, we tried to adopt a per cent value of subclinical reduction in motor skills avoiding an overestimation of hyposmic subjects with possible preclinical PD. Therefore, we opted for a cut-off percentage of worsening higher than the one selected by Horváth et al. ${ }^{13}$ We considered a subclinical idiopathic worsening greater than $15 \%$ in at least 2 motor tasks as a warning sign for preclinical PD.

We carried out a dopaminergic challenge test only in hyposmic subjects, with negative neurological examination, which showed a worsening $>15 \%$ in 2 or more motor parameters (unilaterally or on both sides) over time.

As there are no previous data on the dopaminergic challenge test in the preclinical phase of PD, on the base of clinical reasoning we chose the cut-off percentage of subclinical improvement of motor measurements during dopaminergic stimulus suggestive of a positive response to the test itself.

The clinical reasoning which prompted us to choose this cut-off value is the following: the acute dopaminergic challenge test is generally considered positive, in drug-naïve patients with $\mathrm{PD}$, when an improvement $>20 \%$ is observed. ${ }^{14}$ In a chronic test, the response could even be $>30 \% .{ }^{15}$ Some authors consider this cut-off value too high, especially in very early patients with PD. ${ }^{16}$

Horváth et al $^{13}$ observed that the MICD of MDS-UPDRS III score to detecting improvement is +3.25 points and expressed as 
a percentage is $7.99 \%$ (considering the mean of PD patients' MDSUPDRS III scores $=40.7$ ).

We adopted a percentage cut-off value of improvement at levodopa (L-Dopa) challenge test, which was approximately between the cut-off value in the acute L-Dopa challenge test (20\%) and the percentage of MICD improvement (7.99\%) according to Horváth et al. ${ }^{13}$

A subsequent worsening in motor measurements after stopping L-Dopa may be considered a further sign of a preclinical PD.

\section{1 | Dopaminergic challenge test}

L-Dopa dosage was gradually increased over several days, so that the day before the objective measure of motility (14th), the dose is $200 \mathrm{mg}$ tid and the day of the measurement (15th), the dose is $200 \mathrm{mg}$ of L-Dopa (taken 45 minutes before the test). Then, the dosage was gradually reduced until it was withdrawn.

Objective measurements of movements were conducted at TO before prescribing L-Dopa, at full dopaminergic dosage (T15) and at day 30, when L-Dopa was stopped (T30); (Table S1).

For the evaluation of dopaminergic challenge test, we considered the per cent change of motor measurements at T15 compared with T0 and T30 compared with T15.

An improvement of $>15 \%$ at T15 of the motor measurements which showed a reduction at TO was considered as a positive result; a further worsening of the same parameters at T30 was also considered as a positive result.

Only hyposmic individuals who tested positive at dopaminergic challenge test underwent $\left[{ }^{123} \mathrm{I}\right]-\mathrm{FP} / \mathrm{CIT}$ SPECT.

\section{2 | Additional investigations}

Furthermore, we analysed a control group of healthy subjects, matched by sex and age with the hyposmic subjects. Each subject underwent a first detection of motor tasks (THMF; OPCL; PSUP; TTFCV), which was repeated after 3 days. The results of the second and the first objective measurements were compared (test-retest challenge) using Pearson's linear correlation coefficient.

The comparison of the motor measurements recorded the first detection in the group of hyposmic subjects and in the healthy control group was performed using by $t$ test for independent sample and with Mann-Whitney rank test as appropriate.
The differences between motor performances (Delta value rightleft) of right limbs and the ones of left limbs were recorded, taking into consideration the first measurement in healthy control subjects and the first measurement in hyposmic subjects.

Our study was approved by the Ethical Committee of Massa and Carrara Local Health Institution with the following reference: No. 311, 11/18/2010. Informed consent was obtained from all individual participants included in the study.

\section{3 | INSTRUMENTS}

The wearable devices used for the evaluation and analysis of motor parameters are the inertial sensor systems SensHand v1 and SensFoot v2 (Figure 1), which were appropriately developed for this application. An Inertial Measurement Unit (IMU) integrated in the iNEMO-M1 board based on MEMS sensors (3-axis gyroscope L3G4200D and 6axis geomagnetic module LSM303DLHC) and ARM microcontroller STM32F103RE (STMicroelectronics, Milano, Italy) was used to develop the SensFoot v2 device for lower limbs analysis. The device is placed on the dorsum of subject's foot within an elastic band to ensure the integrality between foot and sensor. SensHand v1, instead, is composed of 4 customized elements with 1 coordination unit included in a bracelet and 3 finger units placed in as many ring packages for thumb, index and middle finger. The bracelet and the 3 rings communicate by means of spiral cables exploiting the Controller Area Network (CAN-bus) standard. Both the controller and the finger boards are equipped with the STM32F103REY6TR High-density ARM-based 32bit Microcontroller (MCU); (STMicroelectronics, Milano, Italy) and a complete 9DOF IMU is composed of the LSM303DLHC e-compass 3D accelerometer and 3D magnetometer module and the L3G4200D triaxial gyroscope (STMicroelectronics, Italy) that guarantee the mapping of motion in the 3-dimensional space.

In both systems, inertial sensor signals are acquired at a frequency rate of $100 \mathrm{~Hz}$. They are supplied by a rechargeable LiPo battery and integrated with Bluetooth module (SPBT2632C2A, v3.0; STMicroelectronics) which wirelessly transmits data acquired to remote personal computer (PC) for offline analysis. The coordination unit synchronizes the data exchange between the nodes of the device and establishes a Bluetooth wireless communication towards a PC station, where a graphical user interface is used by neurologists
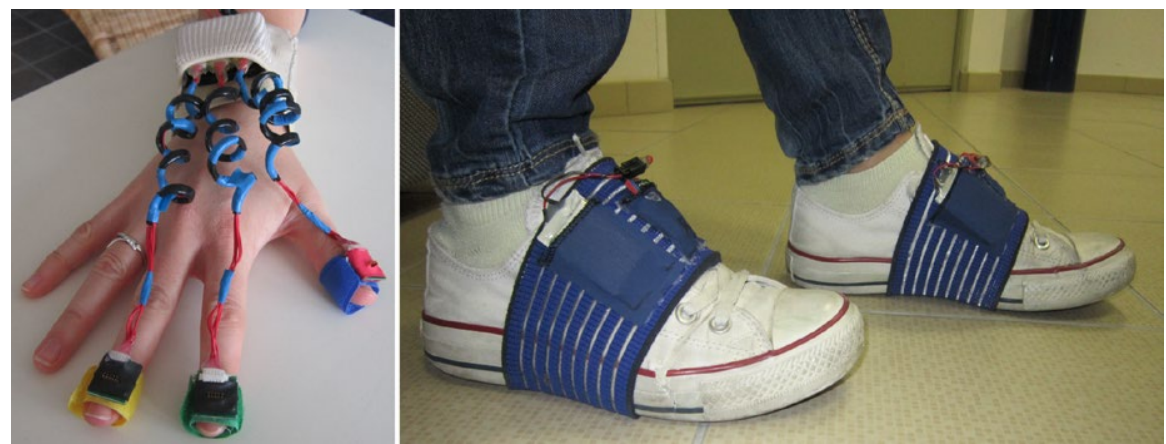
for storing data and to analyse the motion parameters evaluated. SensHand v1 and SensFoot v2 were designed as a small and light structure that is easy to wear and to use, regardless of the physical build of the person wearing it and from variations caused by movement. Preliminary studies on accuracy reveal that these wearable devices have minor discrepancies (coefficient of correlation > 0.9; data not yet published) when the measurements are compared with those obtained with a stereophotogrammetry system (BTS Bioengineering).

Furthermore, the system is not affected by problems related to the deterioration of the sensors and it does not require frequent calibration thanks to the type of sensors, which provide an intrinsic compensation typical of triaxial systems. As for additional properties, the system is characterized by low-cost components and low power consumption as well as long life thanks to low liability to deterioration.

\section{4 | RESULTS}

We screened 287 healthy subjects; 20 of them were chosen because they had idiopathic hyposmia (12 men, 8 women, mean age $67.1 \pm 2.48$ ).

Of 287 subjects selected, we identified 3 cases of mild nasal polyposis and 2 cases of hyposmia following influenza-like infection. These persons have been excluded from our study.
After 1 year, all our hyposmic subjects still had a negative neurological examination. Furthermore, after 1 year, 19 of 20 showed slight motor measurement changes (mean values in Table 1. All the values in Table S2A, B).

One 64-year-old, right-handed and non-smoking male, although still negative at the neurological exam (Video S1), showed a deterioration greater than $15 \%$ in 6 of the 8 parameters analysed (Table 1. All the values in Table S2A, B).

The dopaminergic challenge test with L-Dopa conducted in this subject showed an improvement larger than 15\% in all the 6 parameters that had previously deteriorated at the 1-year follow-up. A new deterioration in these parameters became evident after the discontinuation of the drug (Figure 2. All the values in Table S3).

Eventually the $\left[{ }^{123} \mathrm{I}\right]-\mathrm{FP} / \mathrm{CIT}$ SPECT brain imaging of this subject showed a reduction in the uptake of the ligand bilaterally in the striata, with a prevalence on the right side (Figure 3 ).

A test-retest trial conducted on healthy control subjects showed a good correlation of objective motor measurements obtained in both measurements ( $P$-value < .01) (Tables S4 and S5).

We did not find statistically significant differences in performance between hyposmic subjects and healthy control subjects.

The analysis of the difference in motor performances between right and left limbs showed that healthy subjects performed slightly better with their right limbs (faster movements of the hand and less variability of toe tapping). These results have also been obtained in hyposmic subjects with the exception of a broader variability in toe tapping (Table S6).

TAB LE 1 Motor measurements detected at enrolment and after 1 y in subjects with idiopathic hyposmia

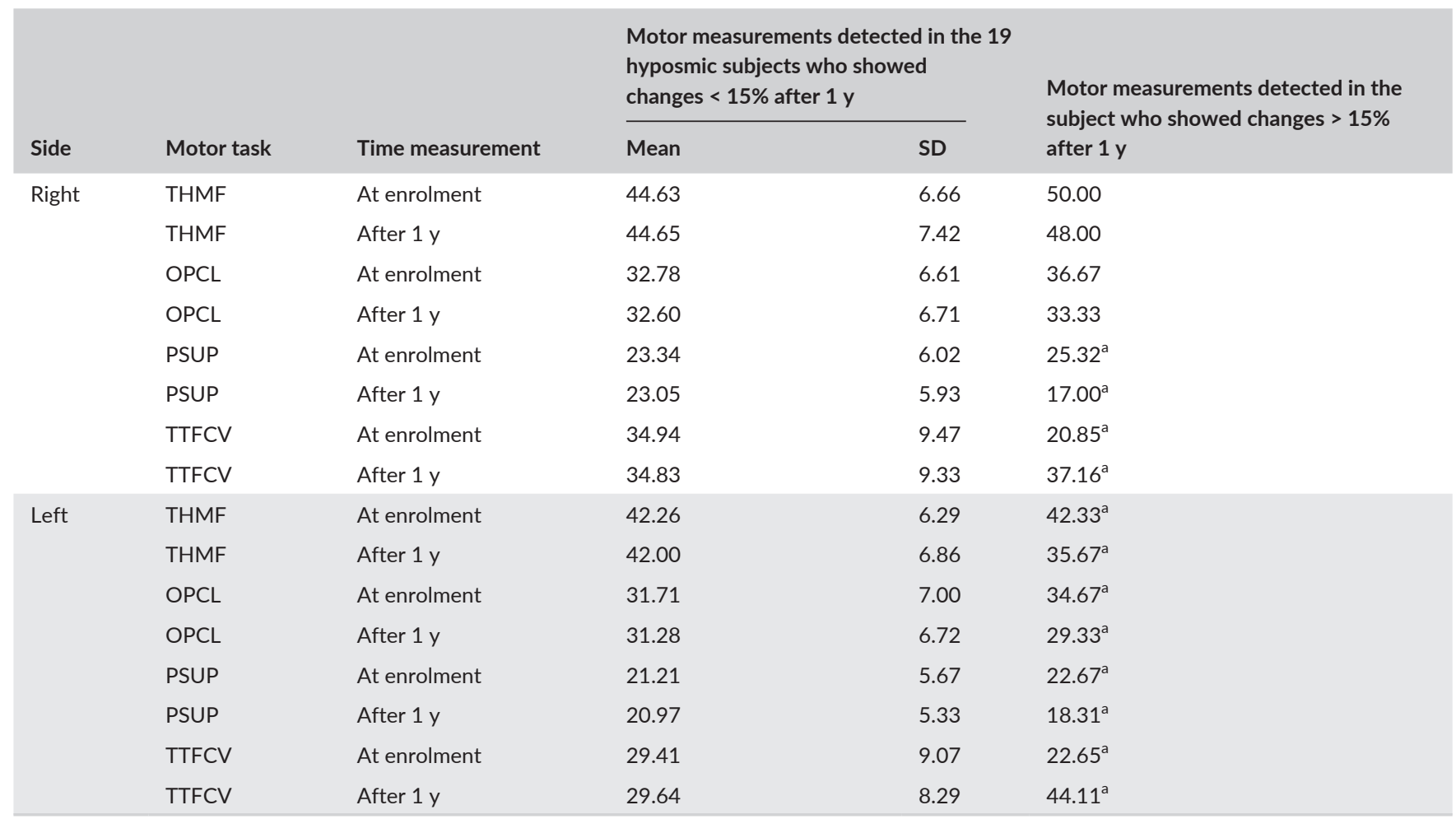

THMF, Thumb-Middle Finger Tapping (number); OPCL, Hand opening-closing (number); PSUP, Hand pronation-supination (number);.TTFCV, Toe Tapping frequency-coefficient of variation: the higher the value less regular is the movement execution.

${ }^{a}$ Motor measurements that showed a per cent variation greater than $15 \%$ at one-year follow-up visit. 
FIGURE 2 Dopaminergic challenge test carried on in the idiopathic hyposmic subject with possible preclinical PD (per cent variation of motor measurements during the different phases of the test)
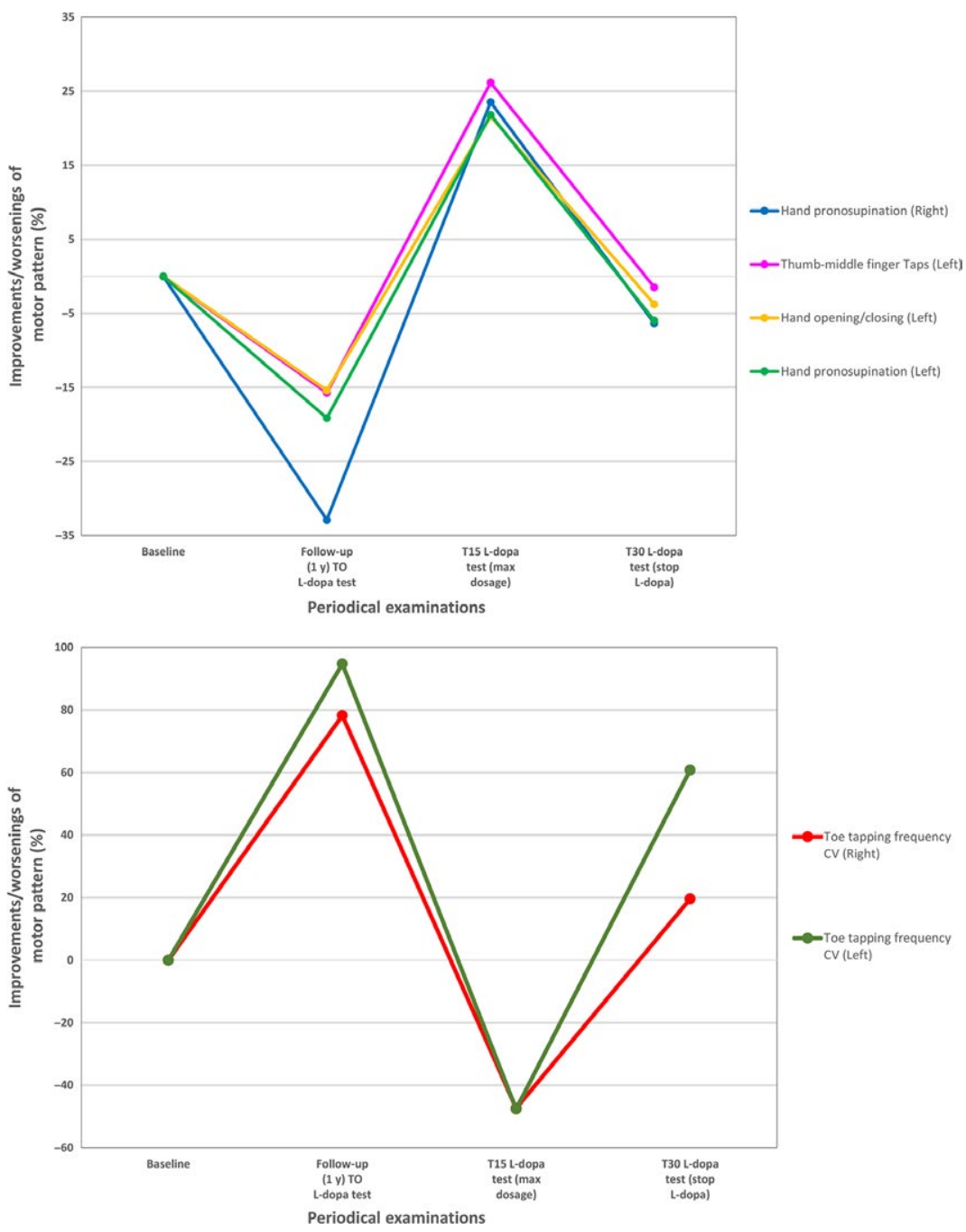

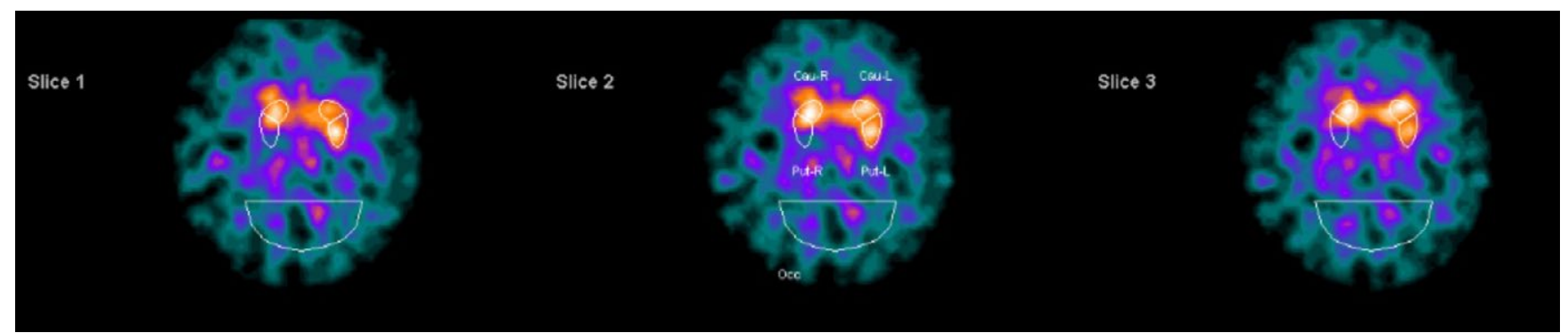

FIGURE $3{ }^{123}$ I-FP-CIT SPECT ( ${ }^{123}$ I-ioflupane) of the subject with possible preclinical PD

\section{5 | DISCUSSION}

Using the inclusion/exclusion criteria adopted for our study, we excluded cases of olfactory dysfunction related to several clinical conditions such as cognitive decline, history of previous concussive head trauma; major otolaryngological, rheumatological, metabolic diseases, malignancies. Indeed, the present study is not meant to estimate the prevalence and causes of olfactory dysfunctions in the general population.
Several devices for the analysis of human motion have been developed in recent years, suitable for objective motor measurements and for discrimination between normal motor patterns and PD patterns. ${ }^{6-10}$ The use of objective measurements for motricity could improve the reliability of motor evaluation of PD patients, reducing the subjective differences that clinical evaluation with motor scales implicate, with evaluation conducted by different operators over time ${ }^{17}$. Nevertheless, the actual use of these tools in daily clinical practice is still limited due to the lack of normative data from the motor 
measurements and of validated clinical scales that use this type of motor measurement.

Normative data and cut-off values for the measurements of motor items in PD evaluations could be very useful in studies on preclinical PD diagnosis: subjects with borderline values in motor measurements could be considered at high PD risk.

Nowadays, it is possible to measure with precision, with wireless technology, certain body movements, among which are those useful for clinical evaluation of patients with PD.

Motor measurements obtained with the sensors used in the present study showed high correlation coefficients in a test-retest proof carried out on a group of healthy control subjects.

We designed a pilot study on preclinical PD diagnosis using objective measurements even in the absence of normative data and cut-off values.

In this study, given the absence of such normative data in literature, we decided to consider every subject with idiopathic hyposmia (therefore at high risk of developing PD) that we assessed as a reference point for himself in the motor measurements conducted over time. We hypothesize that a subject with preclinical PD may have a subtle reduction in motor performances over time until motor abnormalities become clinically evident.

Given the absence of previous references in literature and to choose the degree of subclinical deterioration that might imply a preclinical PD, we deduced from clinical experience a per cent deterioration of motor measurements beyond which would infer preclinical PD (see Materials and Methods). We considered a subclinical idiopathic deterioration greater than $15 \%$ in at least 2 motor tasks as a warning sign for preclinical PD.

A per cent variation $>15 \%$ of motor measurements after 1 year, chosen as cut-off values for motor deterioration after 1 year, cannot be explained with the variability of objective measurements considering also the very low variability within each subject for each measurement (maximum observed SD was 0.44 ) emerged in the rigorous experimental setting.

The question of repeatability of measures can be further studied to a wider range of subjects.

The choice of a cut-off value $>15 \%$ for motor measurements obtained using our precise sensors requires further confirmation on broader populations. Nevertheless, we believe it is a starting point to detect changes over time of motor patterns that could be attributable to biological variations and not only to a mere variability in motor measurements.

We believe that normative data should not be deduced from a small sample like the one shown in our study.

We did not find statistically significant differences in performance between the group of hyposmic subjects and that of healthy control subjects. Perhaps this depends on the small sample of population studied or by the limited number of motor tasks taken into account, but most probably because, as it is well known in literature, ${ }^{5}$ most people with idiopathic hyposmia do not have a preclinical PD.

With broader populations, it will be possible to analyse the role of age, gender, laterality on upper and lower limbs on motor performances, developing solid, normative data.
The lateralization of the motor control should be carefully investigated; in fact, there are several persons who use both hands (or feet) in variable percentage depending on the task they are doing. In addition, lateralization can be discordant in upper and lower limbs.

Subjects in our study, all right-handed, showed better motor performance with right limbs than with left limbs and this is in line with previous literature about finger tapping. ${ }^{18}$

Also, the hyposmic subject with possible preclinical PD demonstrated better performance in tasks using right limbs. Nevertheless, the small sample we analysed does not allow for the establishment of which value of right/left asymmetry could be considered normal and which value could be considered as a cut-off for potential preclinical PD.

Strong normative data would be necessary for using the difference in motor performances between right and left limbs as a parameter in studies on preclinical diagnosis. Possible combinations of laterality in upper and lower limbs should be taken into consideration, as there could be discrepancy between them. Side preference could be variable in different subjects.

With consideration to the side displaying the onset of PD, there could be several possible combinations of motor performance differences between left and right side that might increase or decrease in various ways.

Finally, it cannot be ruled out that in atypical parkinsonism, where bradykinesia is often bilateral, the difference in performance between right and left limbs tends to remain quite stable, despite the disease being slowly progressive.

In our present work, we have adopted a different approach: every subject is a reference for himself for possible changes in motor performances over time.

After a one-year follow-up, only one of the 20 subjects with idiopathic hyposmia showed a subclinical idiopathic deterioration in their motor measurements $>15 \%$. This subject presented a deterioration of all motor indexes measured in left limbs ( 3 on the upper limb and the 1 on the lower) and of 2 motor indexes in right limbs ( 1 on the upper limb and the 1 on the lower). This asymmetry resembles what clinicians usually observe in clinically evident PD.

$\left[{ }^{123} \mathrm{I}\right]-\mathrm{FP} / \mathrm{CIT}$ SPECT is quite invasive and expensive so to limit the number of hyposmic subjects in our population that should undergo this examination, we decided to carry out a dopaminergic challenge test in patients with deterioration of motor measurements over time.

SensHand v1 \& SensFoot v2 ${ }^{6,7}$ inertial sensors were used also to monitor the dopaminergic challenge test.

We opted for an extended dopaminergic challenge test as the acute one often implicates noteworthy side effects. ${ }^{16}$

We evaluated the response to dopaminergic stimulus with a prolonged 15-day test. We did not consider a longer-lasting test to be necessary (chronic test of several months) as the response could be expected within a shorter timescale.

On the basis of clinical reasoning, we chose the per cent cut-off of subclinical improvement of motor measurements, during dopaminergic stimulus, suggestive of a positive response to the test itself (see Materials and Methods). The per cent value of improvement that 
we chose as a cut-off for the dopaminergic challenge test $(>15 \%)$ is arbitrary but reasonable and it needs confirmation within broader populations.

A subsequent deterioration in motor measurements after stopping L-Dopa may be considered a further clue of preclinical PD.

Only the above-mentioned subject underwent a dopaminergic challenge test using wearable devices to monitor the changes in motion parameters.

The pharmacological test showed an improvement greater than $15 \%$ in motor measurements that were previously lowered in this subject and we also observed a deterioration after the discontinuation of L-Dopa. These changes during pharmacological stimulus strengthened the suspect of preclinical PD in this subject.

According to our protocol, it was only this subject that underwent $\left[{ }^{123} \mathrm{I}\right]-\mathrm{FP} / \mathrm{CIT}$ SPECT. The result was in accordance with the hypothesis of an initial neurodegeneration in dopaminergic nigrostriatal pathways. We therefore believe that the subject we identified may have preclinical PD according to $\left[{ }^{123} \mathrm{I}\right]-\mathrm{FP} / \mathrm{CIT}$ SPECT and to the previous screening steps.

In a preceding study by Berendse and Ponsen, ${ }^{5}$ all subjects with idiopathic hyposmia underwent $\left[{ }^{123} \mathrm{I}\right]-\mathrm{FP} / \mathrm{CIT}$ SPECT without further screening tests. All the hyposmic subjects with a positive $\left[{ }^{123} \mathrm{I}\right]-\mathrm{FP} / \mathrm{CIT}$ SPECT developed PD within 5 years. Nevertheless, about $80 \%$ of the hyposmic subjects had negative results in $\left[{ }^{123} \mathrm{I}\right]-\mathrm{FP} / \mathrm{CIT}$ SPECT and did not develop the disease.

According to the same authors, ${ }^{5}$ a good set of preclinical diagnosis should include more screening techniques to be administered for highrisk individuals (ie with idiopathic hyposmia), before considering $\left[{ }^{123} \mathrm{I}\right]$ FP/CIT SPECT. We agree with this vision, and with our pilot study, we tried to develop a good screening set for subjects with idiopathic hyposmia to identify the individuals that should undergo $\left[{ }^{123} \mathrm{I}\right]-\mathrm{FP} / \mathrm{CIT}$ SPECT.

We believe that the subject we identified with our screening set is likely to be affected by preclinical PD. We will only be able to observe whether this individual will develop PD or parkinsonism with further follow-up appointments. We underline some limitations to our work. Cut-off values for suspected preclinical PD, as well as the cut-off values of the dopaminergic challenge test, were chosen by us arbitrarily using logical and clinical suppositions. So, these values need validation in subsequent works with broader populations.

Therefore, because of the arbitrary choice of cut-off values, there may be some of the idiopathic hyposmic subjects with preclinical PD in our population that will not develop a deterioration $>15 \%$ in motor performances in 1 year or, less probably, will not show any deterioration at all. It is possible that the cut-off values we used may cause some "false negatives."

To estimate all the false negatives, all the hyposmic subjects should undergo [123I]-FP/CIT SPECT, but this is not in line with our study protocol. The follow-up of this sample will give some information on this topic over time.

We cannot exclude the fact that the cut-off value we chose could include some "false positives." For example, a subject with idiopathic hyposmia with a deflection of motor measurements greater than $15 \%$ and a positive response to the dopaminergic challenge test $(>15 \%)$ may not always have a positive response to $\left[{ }^{123} \mathrm{I}\right]-\mathrm{FP} / \mathrm{CIT}$ SPECT. Furthermore, a subject who showed a deterioration $>15 \%$ in 1 year could have a negative result in the L-Dopa challenge test, although in this case it may also be atypical parkinsonism. Further studies are necessary to reduce these limitations.

As to the L-Dopa challenge test, the cut-off value we adopted has been chosen following a clinical reasoning. We believe that lowering this value (15\%) could cause an increase in false-positive results.

Smaller L-Dopa dosages could be equally useful in detecting response to the dopaminergic challenge test in subjects with probable preclinical PD. L-Dopa dosage and its administration times could be changed in future studies.

If all subjects with idiopathic hyposmia would undergo a L-Dopa challenge test, maybe we could make a distinction between them on the basis of per cent response at the test, detecting those with possible preclinical PD. But this choice would expose healthy people to a drug, even if for a short period.

For this reason, we preferred a more cautious strategy, proposing a pharmacological test only to hyposmic subjects with a deterioration in motor performance $>15 \%$ in 1 year.

For technical reasons, we have used a limited number of motor measurements. In the lower limbs, we studied the variation in movement frequency instead of its simple frequency, in this way hoping to obtain a more sensible index to detect variations of performances in time. We have to say, however, that this measure was the most variable one. In upper limbs, as we have more measures of frequency of movements, we decided to study the more steady index.

This not homogeneity in adopted measures for upper and lower limbs could be a limit to overcome. So, to use further measurements in a clinical-experimental setting, we must make further efforts to develop new algorithms and improve some important details such as battery life and the autonomy of the instrument. That is why we are aware that a greater number of motor measurements could be used in future studies.

In addition, it is known that "a decrease in the duration of the first step when visual cues are present" ${ }^{19}$ could be a potential predictor of freezing of gait in PD. This parameter, such as the different phases of gait, should be carefully analysed in future studies in the preclinical diagnosis of PD.

The above-mentioned limitations in relation to our study are almost unavoidable according to us, as ours is a pilot study in a new field of research: the preclinical diagnosis of PD. We believe that this field will be constantly updated. ${ }^{2-5}$

In the future, other variables may be taken into consideration in the selection of subjects at risk of developing PD. Several patients in the early phase of PD have a mild cognitive impairment ${ }^{20}$; therefore, a subject with idiopathic hyposmia and $\mathrm{MCl}$ could be at a higher risk of PD and thus be seen as a possible candidate to be monitored over time for motor measurements.

To conclude, over time many improvements may be added, but we believe that the set of screening tests we adopted in this pilot study (olfactory test, motor measurements carried out with inertial sensors 
with their follow-up, and a dopaminergic challenge test monitored with the same sensors) is suitable to detect those hyposmic subjects who should undergo an expensive and invasive test like [123I]-FP/CIT SPECT with a high likelihood of a positive result.

In this study, we do not conclusively demonstrate the validity of our work hypothesis in diagnosing preclinical PD, as this was not the aim of this pilot study. However, the emerging results in this pilot study strongly support the idea that the combined use of an olfactory test and sensors for motion analysis could be very useful for screening aimed at identifying individuals with preclinical PD.

\section{ACKNOWLEDGEMENTS}

We have no acknowledgements to declare.

\section{CONFLICT OF INTEREST AND SOURCES OF FUNDING STATEMENT}

The authors declare competing financial interests. One of the authors (Carlo Maremmani) has filed an Italian patent concerning the IOIT. The Azienda USL Toscana Nordovest and the Scuola Superiore Sant'Anna have filed an Italian patent and an european patent concerning SanHandV1.

\section{ORCID}

C. Maremmani (iD http://orcid.org/0000-0001-8229-2358

\section{REFERENCES}

1. Schapira AH, Tolosa E. Molecular and clinical prodrome of Parkinson disease: implications for treatment. Nat Rev Neurol. 2010;6:309-317.

2. Mahlknecht P, Seppi K, Poewe W. The Concept of Prodromal Parkinson's Disease. J Parkinsons Dis. 2015;5:681-697.

3. Berg D, Postuma RB, Adler $\mathrm{CH}$, et al. MDS research criteria for prodromal Parkinson's disease. Mov Disord. 2015;30:1600-1611.

4. Gaenslen A, Wurster I, Brockmann K, et al. Prodromal features for Parkinson's disease-baseline data from the TREND study. Eur $J$ Neurol. 2014;21:766-772.

5. Berendse HW, Ponsen MM. Diagnosing premotor Parkinson's disease using a two-step approach combining olfactory testing and DAT SPECT imaging. Parkinsonism Relat Disord. 2009;15(Suppl 3):S26-S30.

6. Cavallo F, Esposito D, Rovini E, et al. Preliminary evaluation of SensHand V1 in assessing motor skills performance in Parkinson disease. IEEE Int Conf Rehabil Robot. 2013;2013:6650466.

7. Maremmani C, Bongioanni P, Cavallo F, et al. Preliminary evaluation of Sensorfoot V1 and Senshand V1 in assessing motor skills performance of Parkinson's disease patients. J Neurol Sci. 2013;333(Suppl 1):e67.

8. Rovini E, Esposito D, Maremmani C, Bongioanni P, Cavallo F. Empowering Patients in Self-Management of Parkinson's Disease through Cooperative ICT Systems. In: Morsi YS, Shukla A, Rathore CP, eds. Optimizing Assistive Technologies for Aging Populations. Hershey, PA: Medical Information Science Reference; 2016:251-277.

9. Maetzler W, Domingos J, Srulijes K, Ferreira JJ, Bloem BR. Quantitative wearable sensors for objective assessment of Parkinson's disease. Mov Disord. 2013;28:1628-1637.

10. Ossig C, Antonini A, Buhmann C, et al. Wearable sensor-based objective assessment of motor symptoms in Parkinson's disease. J Neural Transm (Vienna). 2016;123:57-64.

11. Folstein MF, Folstein SE, McHugh PR. "Mini-mental state". A practical method for grading the cognitive state of patients for the clinician. $J$ Psychiatr Res. 1975;12:189-198.

12. Maremmani C, Rossi G, Tambasco N, et al. The validity and reliability of the Italian Olfactory Identification Test (IOIT) in healthy subjects and in Parkinson's disease patients. Parkinsonism Relat Disord. 2012;18:788-793.

13. Horváth $\mathrm{K}$, Aschermann Z, Ács $\mathrm{P}$, et al. Minimal clinically important difference on the Motor Examination part of MDS-UPDRS. Parkinsonism Relat Disord. 2015;21:1421-1426.

14. Albanese A, Bonuccelli U, Brefel C, et al. Consensus statement on the role of acute dopaminergic challenge in Parkinson's disease. Mov Disord. 2001;16:197-201.

15. Gilman S, Wenning GK, Low PA, et al. Second consensus statement on the diagnosis of multiple system atrophy. Neurology. 2008;71:670-676.

16. Reichmann H. Clinical criteria for the diagnosis of Parkinson's disease. Neurodegener Dis. 2010;7:284-290.

17. Post B, Merkus MP, de Bie RM, de Haan RJ, Speelman JD. Unified Parkinson's disease rating scale motor examination: are ratings of nurses, residents in neurology, and movement disorders specialists interchangeable? Mov Disord. 2005;20:1577-1584.

18. Hubel KA, Reed B, Yund EW, Herrn TJ, Woods DL. Computerized measures of finger tapping: effects of hand dominance, age and sex. Percept Mot Skills. 2013;116:929-952.

19. Chong RK, Lee K-H, Morgan J, Wakade C. Duration of step initiation predicts freezing in Parkinson's disease. Acta Neurol Scand. 2015;132:105-110.

20. Pellecchia MT, Picillo M, Santangelo G, et al. Cognitive performances and DAT imaging in early Parkinson's disease with mild cognitive impairment: a preliminary study. Acta Neurol Scand. 2015;131:275-281.

\section{SUPPORTING INFORMATION}

Additional Supporting Information may be found online in the supporting information tab for this article.

How to cite this article: Maremmani C, Cavallo F, Purcaro C, et al. Combining olfactory test and motion analysis sensors in Parkinson's disease preclinical diagnosis: a pilot study. Acta Neurol Scand. 2017;00:1-8. https://doi.org/10.1111/ ane.12862 